\title{
The double benefit of Spalax p53: surviving underground hypoxia while defying lung cancer cells in vitro via autophagy and caspase-dependent cell death
}

\author{
Martin Ellis ${ }^{1,2}$, Orly Stern ${ }^{1}$, Osnat Ashur-Fabian ${ }^{1,2,3}$ \\ ${ }^{1}$ Translational Hemato-Oncology Laboratory, Hematology Institute and Blood Bank, Meir Medical Center, Kfar-Saba, 4428164, \\ Israel \\ ${ }^{2}$ Sackler Faculty of Medicine, Tel Aviv University, Tel Aviv, 69978, Israel \\ ${ }^{3}$ The Department of Human Molecular Genetics and Biochemistry, Sackler Faculty of Medicine, Tel Aviv University, Tel Aviv, \\ 69978, Israel
}

Correspondence to: Osnat Ashur-Fabian, email: osnataf@gmail.com

Keywords: spalax, p53, lung cancer, autophagy, caspases

Received: June 09, 2016

Accepted: August 15, 2016

Published: August 20, 2016

\section{ABSTRACT}

The blind subterranean mole rat, Spalax ehrenbergi, is a model organism for hypoxia tolerance. This superspecies have adapted to severe environment by altering an array of hypoxia-mediated genes, among which an alteration in the p53 DNA binding domain (corresponding to R174K in humans) that hinders its transcriptional activity towards apoptotic genes. It is well accepted that apoptosis is not the only form of programmed cell death and that mechanisms that depend on autophagy are also involved. In the current work we have extended our research and investigated the possibility that Spalax p53 can activate autophagy. Using two complementary assays, we have established that over-expression of the Spalax p53 in p53-null cells (human lung cancer cells, H1299), potently induces autophagy. As Spalax is considered highly resistant to cancer, we further studied the relative contribution of autophagy on the outcome of $\mathrm{H1299}$ cells, following transfection with Spalax p53. Results indicate that Spalax p53 acts as a tumor suppressor in lung cancer cells, inducing cell death that involves autophagy and caspases and inhibiting cell number, which is exclusively caspase-dependent. To conclude, the Spalax p53 protein was evolutionary adapted to survive severe underground hypoxia while retaining the ability to defy lung cancer.

\section{INTRODUCTION}

The blind subterranean mole rat, Spalax ehrenbergi superspecies, is a model organism for hypoxia tolerance. Spalax, has an exceptionally long life-span, spent entirely in underground sealed burrows under extreme hypoxic conditions [1,2]. It survives $6 \% \mathrm{O}_{2}$ and $7 \% \mathrm{CO}_{2}$ in nature, and $3 \% \mathrm{O}_{2}$ and up to $15 \% \mathrm{CO}_{2}$ for approximately $11 \mathrm{~h}$ in laboratory settings, whereas Rattus die after $2-4 \mathrm{~h}$ in similar conditions [3].

p53 is a master gene orchestrating an array of tumor suppressing activities in response to a variety of stress conditions $[4,5]$, including hypoxia. p53 is among the most mutated proteins in human cancers. The Spalax p 53 binding domain was shown to contain a specific amino acid substitution (corresponding to R174K in humans) with a bias against the transcription of apoptotic genes while favoring cell arrest and DNA repair genes [6]. This mechanism is believed to contribute to Spalax's hypoxia adaptation by escaping from apoptosis [6-9].

It is now well accepted that apoptosis is not the only form of programmed cell death and that mechanisms that depend on autophagy are also implicated $[10,11]$. Thus, we were specifically interested to explore the ability of the Spalax p53 to induce autophagy. Using two complementary autophagy assays, we have established that Spalax p53 is able to potently activate autophagy in the p53-null human lung cancer cells (H1299). As the blind mole rat is highly cancer resistant [12], we were further interested to explore the whether the mechanisms that Spalax have evolved in the $p 53$ gene to survive hypoxia, might have an advantage relating to cancer 
resistance. Our results established that Spalax p53 acts as a tumor suppressor, inhibiting H1299 cell number that is exclusively caspase-dependent, while inducing cell death that involves both autophagy and caspases. To the best of our knowledge this is the first demonstration of such an activity by the Spalax p53 protein, which was evolutionary adapted to survive severe underground hypoxia while retaining the ability to defy cancer.

\section{RESULTS}

\section{Spalax p53 activates autophagy in lung cancer cells}

We have previously shown that Spalax p53 evolves a substitution in the DNA binding domain that hinders its transcriptional activity towards apoptotic genes [6, 7]. In the current work we have extended our research and investigated the possibility that Spalax p53, retained the ability of the human p53 [13] to activate autophagy.

The extent of autophagy was studied in the p53null human non small cell lung cancer model (H1299), a valuable platform for researching p53-related activities. Spalax p53 plasmids were transiently transfected into the cells. Human wild type p53 plasmid was used for comparison. The cells were stained, 72 hours post transfection, with the lysosomotropic agent, acridine orange. This dye accumulates in acidic compartment and is used to detect and quantify acidic vesicular organelles (AVO), characteristic of autophagy activation. This accumulation is observed as a bright red fluorescence, which is proportional to the degree of authophagy in the cells. For normalization, transfection with appropriate empty vectors (pCMV for the human p53 or pCDNA3 for the Spalax p53), were used (Supplementary Figure S1). Fluorescence was recorded by a fluorescence microscope equipped with a camera and quantified using NIH ImageJ software. Results (Figure 1A) have indicated a low level of basal authophagy in the non-transfected cells, which was induced by 3.9-fold following transfections with the Spalax p53 plasmid. The human p53 induced 3.6-fold increase in autophagy, while a 3.3-fold was observed by the positive control, $3 \%$ hydrogen peroxide $\left(\mathrm{H}_{2} \mathrm{O}_{2}\right)$. The experiments were further conducted in the presence of an autophagy inhibitor, Bafilomycin A1, which effectively reversed autophagy induction in all cases, indicating specificity. Representative fluorescent microscopy images are presented in Figure 1B.

Next, the effect of Spalax p53 on authophagy in the cells was further evaluated by measuring the turnover of intra-cellular GFP-LC3. The change in GFP levels reflects an autophagic flux and is used as an indicator of cellular autophagic activity in living cells. Increased autophagic flux is expected to result in a progressive delivery of GFPLC3 to autolysosome, where this substrate undergoes degradation. Therefore, enhanced autophagic flux is detected as a decrease in total cellular GFP signal. H1299 cells were transfected with Spalax p53 plasmid in the presence or absence of GFP-LC3. Human wild-type p53 was used for comparison. As controls, cells tranfected with GFP-LC3 alone or co-transfected with appropriate empty vectors (pCMV or pCDNA3), were used (Supplementary Figure S2). Changes in total intracellular GFP-LC3 fluorescence intensity were measured 72 hours post transfection by a MACSQuant flow cytometer. Mean Fluorescence Intensity (MFI) for each treatment was determined and is presented in Figure 2A. Compared to transfection with the empty vectors, a $30 \%$ decrease in GFP signal intensity was observed in H1299 cells transfected with the Spalax p53. Using this method, transfection with the human wild-type p53 resulted in a $60 \%$ decrease in GFP intensity. The difference between the Spalax and human p53's were significant $(p<0.05)$. Representative flow cytometer histograms are presented for each treatment (Figure 2B). Taken together, we have demonstrated, by two complementary methods, albeit with different potencies in comparison to the human p53, that Spalax p53 is able to induce authophagy in human lung cancer cells.

\section{Spalax p53 induces lung cancer cell death that involves authophagy and caspases}

The novel observation that Spalax p53 is able to induce autophagy in lung cancer cells, led us to explore the involvement of autophagy in an array of cellular activities. H1299 cells were transfected for 72 hours with expression plasmids for Spalax p53. Human wild type or mutated p53 (H179R), served for comparison. Transfections with empty vectors, pCMV for human and mutated, and pCDNA3 for Spalax p53, were used for normalization (Supplementary Figure S3). In order to investigate the relative contribution of autophagy, 3-MA, an autophagy inhibitor, was used. In parallel, dependence on caspases was assessed using the pancaspase apoptosis inhibitor Z-VAD-FMK. First, the extent of cell death was determined by the Annexin-PI assays.

Representative Annexin-PI results, presented in Figure 3A, indicate that Spalax p53 induced a 2.7-fold increase in cell death. This cell death was prevented by both pathway inhibitors, 3MA and Z-VAD-FMK. This result provides the first indication that cell death caused by Spalax p53 in lung cancer cells is not only caspasedependent but also involves autophagy. In contrast, the 2.3 -fold increase in cell death by the human p53 was effectively rescued only in the presence of Z-VAD-FMK and was not influenced at all by treatment with $3 \mathrm{MA}$. The difference between the Spalax and human p53's were significant $(p<0.05)$. Representative Annexin-PI results (Figure 3B) indicate that Spalax p53, comparable with the human p53, induced early apoptosis (Annexin+/PI-) and, more profoundly, late apoptosis/necrotic (Annexin+/PI+) cell death. 
The effect of the Spalax p53 on cell death was further assessed by measuring SubG1 fraction, indicative of apoptotic cell death, using cell cycle analysis. As detailed above, H1299 cells were transfected with Spalax p53 in the presence/absence of Z-VAD-FMK or 3MA. After 72 hours of incubation, cell cycle was analyzed by flow cytometry. Analysis following transfections with wild type human p53 or H179R mutated p53 plasmids served as controls. Appropriate empty vectors, in the presence/ absence of both inhibitors, were used for normalization (Supplementary Figure S4). Results from a representative experiment are shown. A basal 15\% SubG1 fraction, indicative of apoptotic cell death (Figure 4A), was documented in the non-transfected cells, which was inhibited in the presence of both Z-VAD-FMK and 3MA. The SubG1 cell fraction was significantly induced by the Spalax p53 (3-fold). Comparable with the AnnexinPI results, this effect was effectively reversed by both 3MA and Z-VAD-FMK. The human p53 induced a more potent increase in subG1 (3.5-fold) which, similar to the
Annexin-PI results, was blocked by Z-VAD-FMK, but not 3MA. No significant difference was observed between the two p53's and no effect was observed by the mutated p53. Collectively our findings highlight that while the human p53 regulates lung cancer cell death exclusively via caspase-dependence, Spalax p53 induction of cell death appears to involve authophagy-dependent mechanism as well as caspases. A representative cell cycle experiment, depicting the subG1 percentage in all treatments, is shown in Figure 4B.

\section{Inhibition of lung cancer cell proliferation by the Spalax p53 is caspase-dependent}

Lastly, similar to wild type human p53, lower cell density in the presence of Spalax p53 was documented, while higher cell density was shown in the presence of the mutated p53 (Figure 5A). These results inspired us to further examine the role of Spalax-p53-induced autophagy on cell proliferation. H1299 cells were transfected with

\section{A $\quad$-Bafilomycin A +Bafilomycin A}

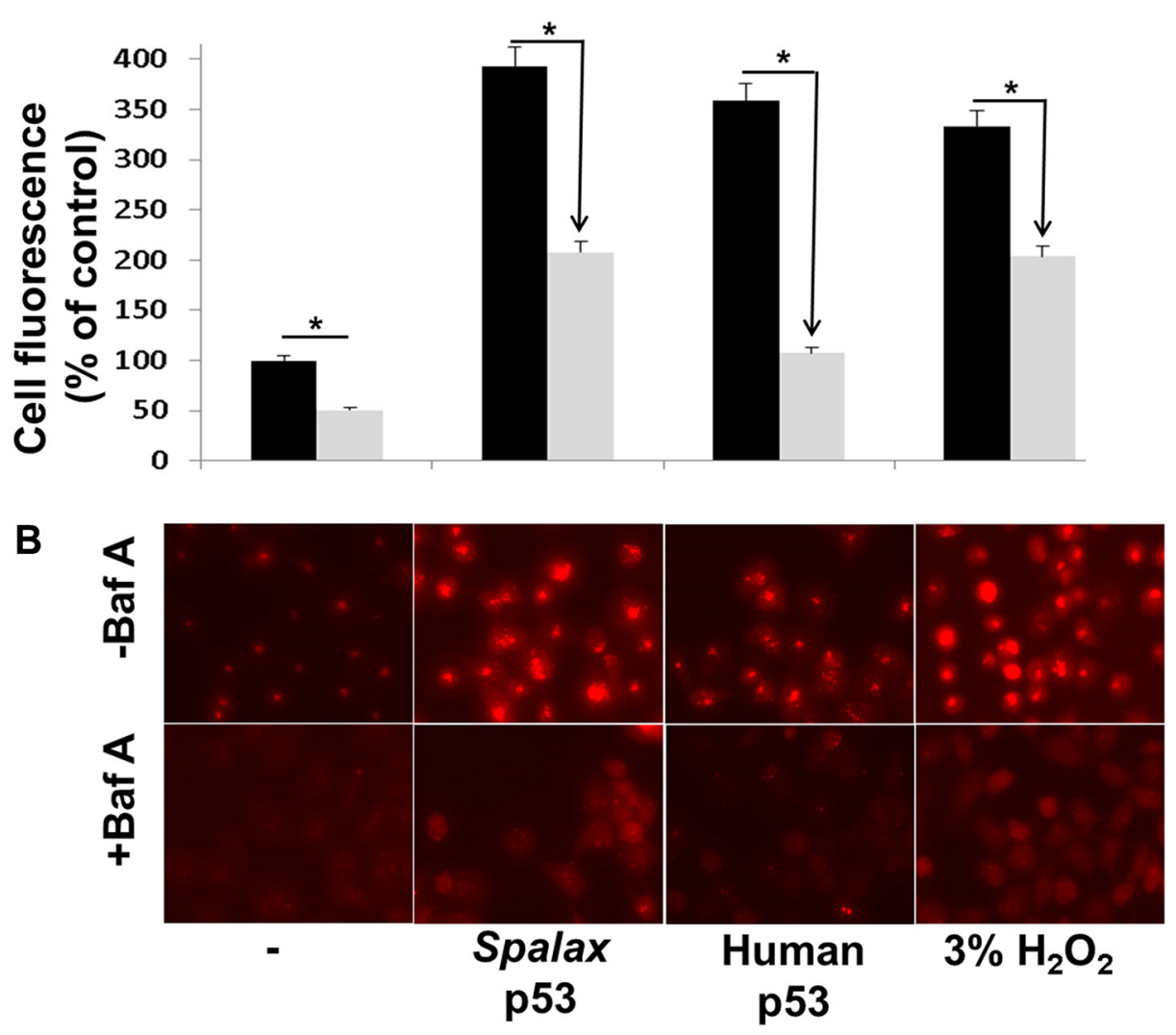

Figure 1: Human and Spalax p53 initiate authopagy in lung cancer cells. H1299 cells were transfected with the human or Spalax p53 plasmids for 72 hours, after which the cells were stained with acridine orange. Fluorescence in four fields per well were counted by fluorescence microscope equipped with a camera and an average value was calculated using NIH ImageJ software. 3\% hydrogen peroxide $\left(\mathrm{H}_{2} \mathrm{O}_{2}\right)$ was used as positive control. The experiments were conducted in the presence and absence of an autophagy inhibitor, Bafilomycin A1 (Baf A). (A) Cell fluorescence (\% of control) for the different treatments. (B) Representative fluorescent microscopy images. Experiments were repeated twice. Results are presented as \% of empty vectors (pCMV for the human wild type p53; pCDNA3 for the Spalax $553{ }^{*} p<0.05$ 
expression plasmids for Spalax p53, in the presence or absence of a 3MA or Z-VAD-FMK. Human wildtype p53 or a mutated form of the human p53 (H179R), served as a positive or negative controls, respectively. Appropriate empty vectors, pCMV for human and pCDNA3 for Spalax p53, were used in each experiment for normalizations. After 72 hours of incubation, absolute cell counts and cell proliferation were evaluated. In the non-transfected cells, an increase in cell number (Figure 5B) was observed in the presence of both Z-VAD-FMK or 3MA, indicating involvement of both apoptosis and autophagy on cell number in this cancer cell model. In the presence of a plasmid containing a human mutated p53, a 2.8-fold increase in cell number was documented. While wild type human p53 inhibited the number of cells by $43 \%$, Spalax p53 had a superior effect, resulting in a potent reduction of $72 \%$ in cell number. However, the difference between the two p53's did not reach statistical significance. The inhibitory effect of Spalax p53 on cell number was significantly reversed in the presence of Z-VAD-FMK, but not by 3MA. WST-1 proliferation assay (Figure 5C) further confirmed that Spalax p53 reduced cell proliferation by $34 \%$ and the human p53 by $28 \%$. This reduction, comparable with the cell counts, was rescued for both p53's by Z-VAD-FMK, but remained unaffected by the autophagy inhibitor, 3MA. These results suggest that the regulation of cell proliferation in H1299 lung cancer cells by the Spalax p53 is exclusively mediated via caspase-dependent mechanisms and does not involve activation of autophagy.

\section{DISCUSSION}

Cells exposed to hypoxic conditions activate wildtype p53 protein, resulting in apoptotic cell death. Hypoxia was suggested as a selection pressure, frequently mutating p53 in tumors, abrogating hypoxia-induced apoptosis. Spalax is a blind mole rat that spends its life underground, living, mostly during winter floods, under extreme hypoxic conditions. This superspecies have adapted to severe environment by altering an array of hypoxiamediated genes [3, 14-18]. By cloning the Spalax p53 mRNA a Spalax-specific modification was revealed within the p53 DNA binding domain, replacing arginine $(\mathrm{R})$ with lysine $(\mathrm{K})$ at position 172 (corresponding to codon 174 in humans). This specific mutation was reported in tumors of various types, suggesting that the evolution of p53 in hypoxia-stressed Spalax mimics human tumor evolution [6]. By using reporter assays, it was further discovered that the Spalax p53 protein is transcriptionally biased toward G1 arrest activation and impaired for apoptosis target genes, of both human [6] and Spalax [7] origin.

It is now well accepted that apoptosis is not the only form of programmed cell death and that mechanisms

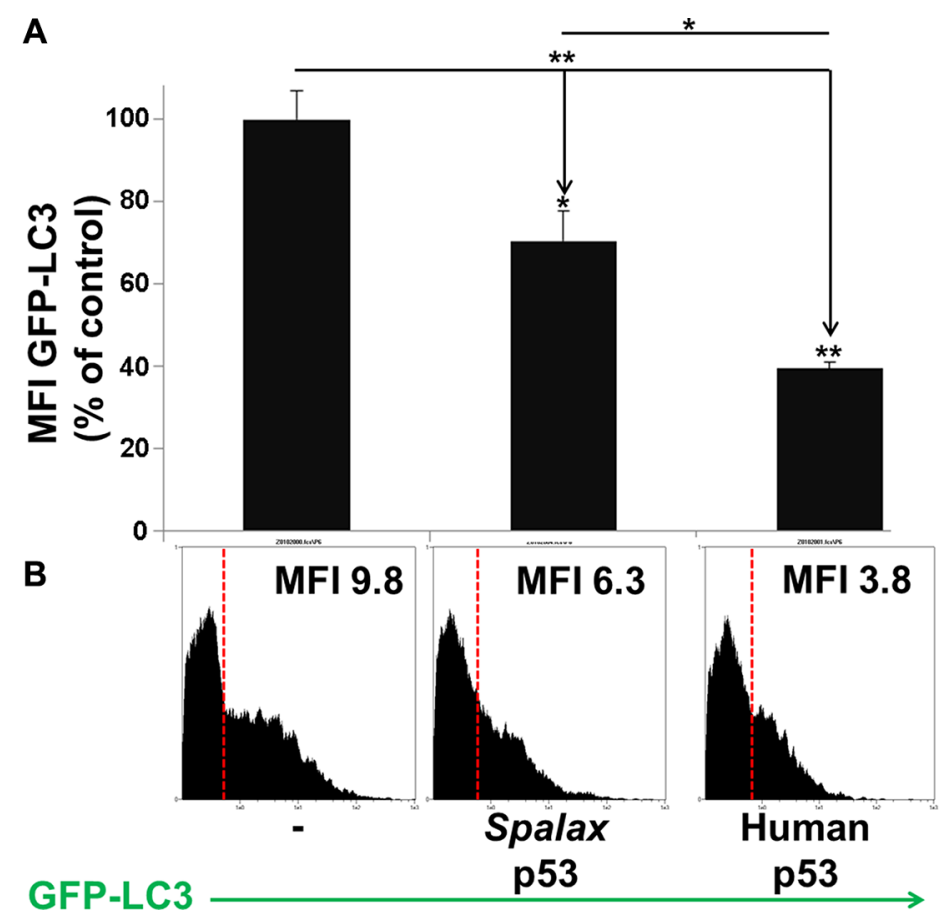

Figure 2: H1299 cells were transfected with the human or Spalax p53 plasmids in the presence or absence of GFP-LC3. As controls, cells tranfected with GFP-LC3 alone or co-transfected with empty vectors (pCMV or pCDNA3), were used. Changes in total intracellular GFP-LC3 fluorescence intensity were measured 72 hours post transfection by flow cytometry. (A) Mean Fluorescence Intensity (MFI) for each treatment is presented. (B) Representative FACS histogram with MFI values for each treatment is depicted. Results were repeated twice, in duplicates. Results are presented as \% of empty vectors (pCMV for the human wild type p53; pCDNA3 for the Spalax p53). ${ }^{*} p<0.05, * * p<0.005$. 
that depend on autophagy are also involved [10]. We were therefore interested to investigate the possibility that Spalax p53 can activate autophagy. Non small cell lung cancer cells, that are p53-null (H1299), served as the cellular platform. Autophagy is characterized by the formation of acidic vesicular organelles (AVO) in the cell cytoplasm [19], leading to disruption of cytoplasm compartments before nuclear collapse. In our study, we have used the supravital stain acridine orange which accumulates in acidic compartment and is proportional to the degree of authophagy in the cells. A second complementary method to elucidate the level of autophagy in the cells was the GFP-LC3 turnover, an indicator for autophagy flux. We have confirmed, by the two methods, albeit with different potencies in comparison to the human p53, that Spalax p53 is able to activate autophagy in the cancer cell model. We have further corroborated the mechanism of Spalax-induced autophagy by using bafilomycin A1, an autophagy inhibitor. Activation of autophagy by the human p53, as shown in this current work, was previously reported [20] and suggests to be an integral part of p53 tumor suppressive function [21]. We were not able to conclude, based on the methods used in this work, whether the extent of autophagy initiated by Spalax p53 is equal, superior, or inferior, compared with the human wild type p53. It was previously shown that in tissues generated from Spalax hearts, under hypoxic conditions, mitophagy, the selective degradation of the mitochondria by autophagy, occurs [22]. Interestingly, in another study, the sub population of Spalax galili, living in chalk soil, showed significantly higher protein levels of the autophagy protein ATG7 and of the measure of autophagic flux, based on the LC3-II/LC3-I ratio [23]. Other underground living and burrowing animals, such as the naked mole rat, showed a similar high level of autophagy markers in multiple tissues [24]. Our report is, to the best of our knowledge, the first to stress the direct capability of the Spalax p53 to induce macroautophagy and is to report such an action in cancer cells in vitro.

In addition to numerous adaptations to underground life, Spalax is characterized by a remarkable longevity, with a maximum documented lifespan of 21 years. Further studies have established that the blind mole rat is highly cancer resistant [12]. Among thousands
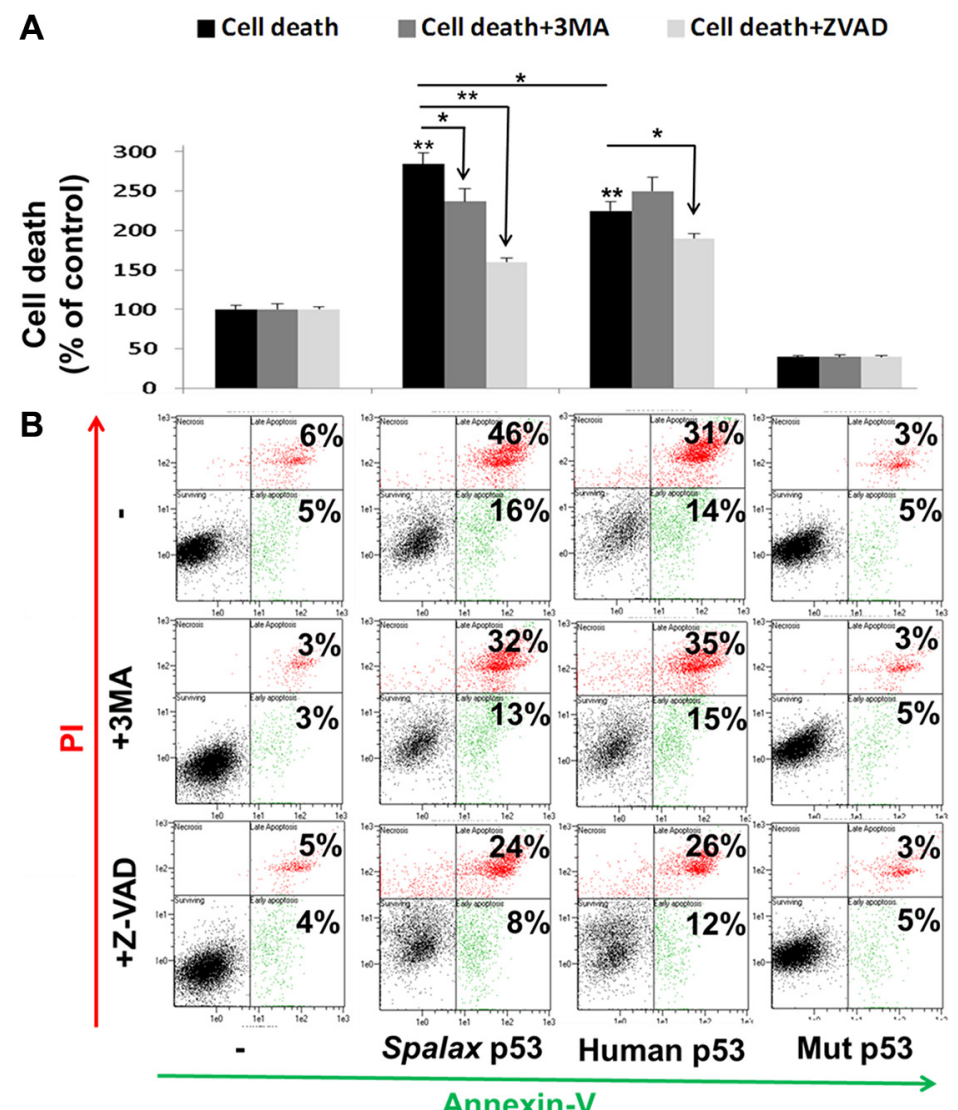

Figure 3: Spalax p53 regulates lung cancer cell death via both autophagy and caspases. H1299 cells were transfected with wild type human p53, Spalax p53 and mutated human H179R p53 (Mut p53) in the presence/absence of an apoptosis inhibitor (Z-VAD-FMK) or authophagy inhibitor (3MA) and examined after 72 hours by Annexin-PI assay (FACS). (A) Late apoptosis cell fraction (\% of control). (B) Representative FACS results. The $\%$ of cells in early (An+/PI-, green) and late apoptosis/necrosis (An+/PI+, red) for each treatment are depicted.The experiments were repeated thrice, in duplicates. Results are presented as \% of empty vectors \pm inhibitors (pCMV for the human wild type and mutated $\mathrm{p} 53$; pCDNA3 for the Spalax $\mathrm{p} 53) .{ }^{*} p<0.05,{ }^{*} p<0.005$. 
of captive animals over a 40-year period, not even a single case of spontaneous tumor have been developed, including in over 20 years old animals that may be more vulnerable to cancer. Moreover, chemical carcinogens failed to induce tumor growth in Spalax, while tumors were clearly evident in other rodents and primary Spalax fibroblast inhibited growth and killed cancer cells, but not normal cells, either through direct fibroblast-cancer cell interaction or via soluble factors [12]. Yet, the effect of Spalax p53 on the fate of cancer cells is a research direction that was largely overlooked. This is since early studies of the Spalax p53 primarily aimed to evaluate the transcription factor activities of this specific p53 protein [6]. Consecutive studies have further explored the transcription of additional target genes by the Spalax p53 under normoxic versus hypoxic environment [7, 25-27]. We were therefore interested to study the direct anticancer effect of the Spalax p53. Such anti-cancer effects by wild type human p53 [28-30] and the mitogenic effects by codon 179 mutated p53 [28, 31] in H1299 cells correspond with our current results. We have further confirmed that Spalax p53 can significantly inhibit lung cancer cell proliferation and induce potent cell death in vitro. Support that the Spalax p53, that was shown to be transcriptionally biased against apoptotic target genes, is able to induce cell death in vitro, was previously demonstrated by our group in a murine pro $\mathrm{B}(\mathrm{Ba} / \mathrm{F} 3)$ cell line [7]. In this leukemia cell model, Spalax p53 was less potent in inducing SubG1, compared to human p53. Similarly, in this current work, in the non-small cell lung cancer cells, a solid tumor model, induction of SubG1 by the Spalax-p53 was somehow lower compared with the effect by the human p53. However when we analyzed the extent of cell death by another methods, Annexin-PI, the impact of spalax p53 on cell death was higher compared to human wild type p53. The discrepancy between the two methods (cell cycle and Annexin-PI) may be explained by the observations that phosphatidylserines externalization and binding of Annexin-V may occur not only in apoptosis, but also in other types of cell death including necrosis [32] and autophagy [33-35]. Therefore while SubG1 analysis by cell cycle is indicative only for apoptotic cell death, positive signal with Annexin V staining can be considered a marker of any type of cell death and may provide additional valuable information [36]. In another recent study, $S$. galili p53, sibling species with the $S$. judaei, failed to induce early apoptotis (Annexin+/PI-) in H1299. Nonetheless, in this experiment the cells were

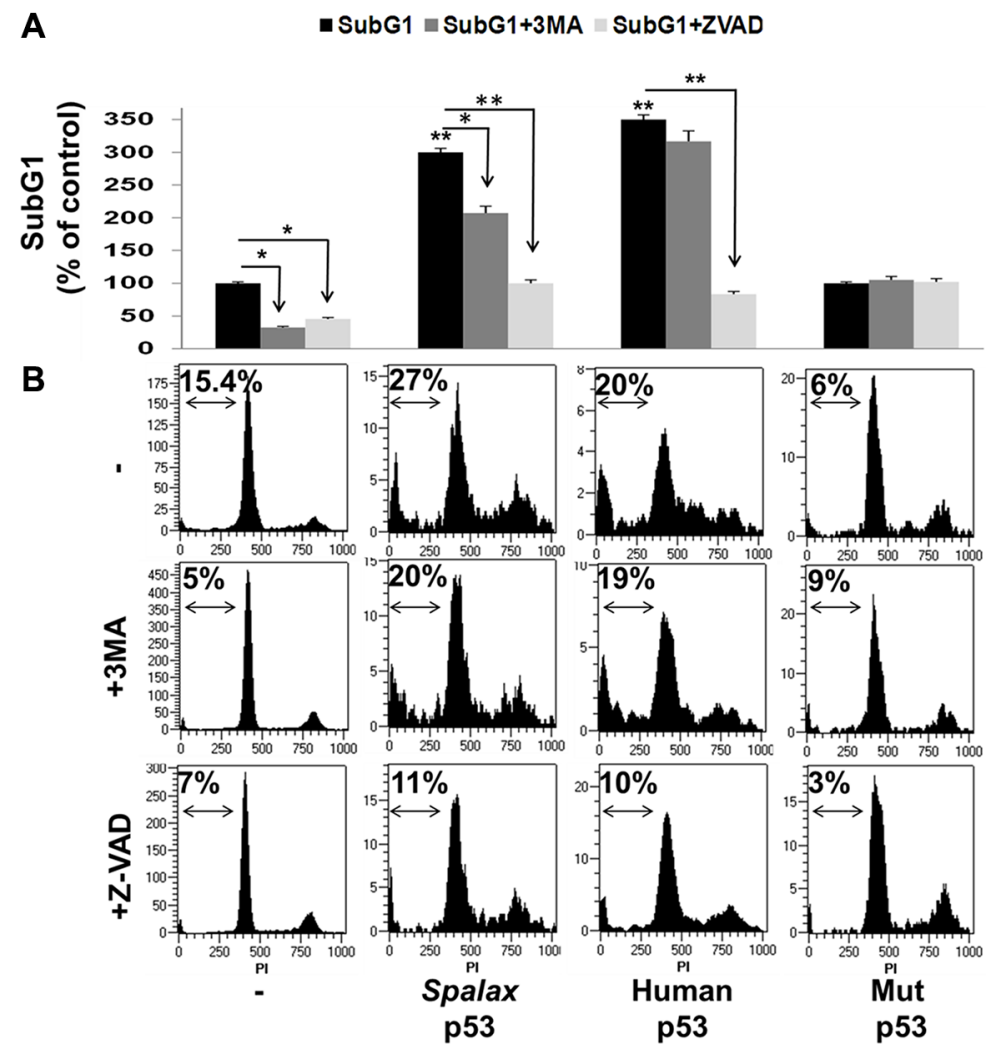

Figure 4: Spalax 53 regulates lung cancer SubG1 via both autophagy and caspases. H1299 cells were transfected with wild type human p53, Spalax p53 and mutated H179R human p53 (Mut p53) in the presence/absence of an apoptosis inhibitor (Z-VAD-FMK) or authophagy inhibitor (3MA) and (A) apoptotic cell fraction (SubG1) was examined by cell cycle (FACS) after 72 hours (B) Representative FACS results. The $\%$ of SubG1 cells for each treatment is depicted. The experiments were repeated thrice, in duplicates. Results are presented as $\%$ of empty vectors (pCMV for the human wild type and mutated p53; pCDNA3 for the Spalax p53) \pm inhibitors. ${ }^{*} p<0,05$, $* * p<0.005$. 
analyzed 24 hours post transfections, and not 72 hours as in our study and no data regarding late apoptosis (Annexin+/PI+), which was the main cell death observed in our system, was presented. In addition, although the S. galili and the S. judaei p53 share the same protein sequence, carrying an R172K mutation, there are two synonymous variations between the two species [9]. The ability of p53 of other mammals of the Tibet plateau, the wild zokor (Myospalax baileyi) and root vole (Microtus oeconomus), to induce apoptosis in cervical cancer cells, was demonstrated as well [37].

Lastly, based on our observation that the Spalax p53 can activate autophagy, we were particularly interested to assess the relative contribution of autophagy versus caspases on cancer cell proliferation and death. By using an autophagy inhibitor (3MA) or a pan caspase inhibitor (Z-VAD-FMK), it emerged that inhibition of cell proliferation by the Spalax p53, similar to the human p53, is solely caspase-dependent. In contrast, while the induction of cell death by the human p53 was utterly caspase-mediated, the death induced by the Spalax p53 had a mixed phenotype of autophagy and caspase-dependence. These results propose that there is a distinction between the two p53's with regards to the mechanisms through which cancer cell death is promoted.
The observation that the inhibition of autophagy rescued cancer cells from death induced by the Spalax p53 provided the first evidence to substantiate that by activation of the autophagic pathway, Spalax p53 may directly eliminate cancer cells. It is well established that elimination of cancer cells may occur not only via apoptosis but could also be mediated by autophagydependent mechanisms. Support for that came from studies in which a decline in autophagic activity was related to tumorigenesis, whereas autophagy activation led to cancer cell death $[10,19,20,38-40]$. We have therefore hypothesized that, as part of the cancer resistance phenotype, Spalax p53 may be efficient in inducing cancer cell death that is autophagy, as well as caspase-dependent. It is now clear that caspases are a molecular switch node in the crosstalk between autophagy and apoptosis [41, 42].

Similarly, human p53-induced-autophagy was recently shown to contribute to the execution of $\mathrm{p} 53$ dependent apoptosis, indicating that p53 can promote cell death through multiple pathways [13]. Indeed, autophagy and apoptosis often occur in the same cell, mostly in a sequence in which autophagy precedes apoptosis [43-45]. These observations are in accord with a recent report indicating that autophagy can lead to the execution of apoptotic (type I) or necrotic (type III) cell death [39],
A
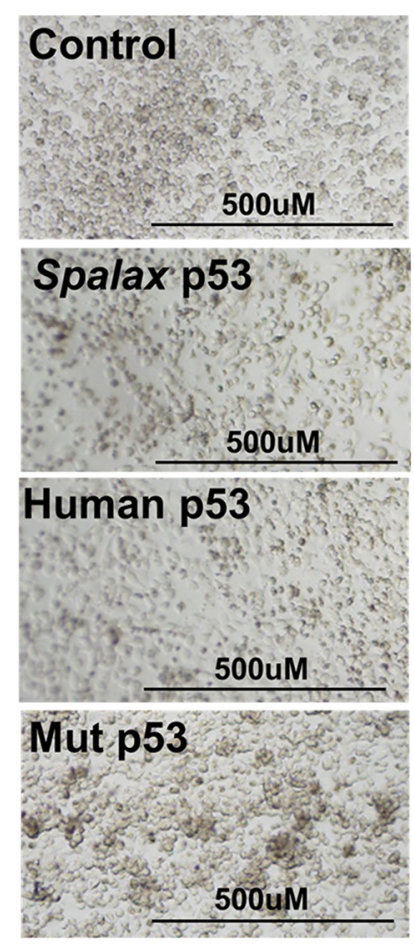

B

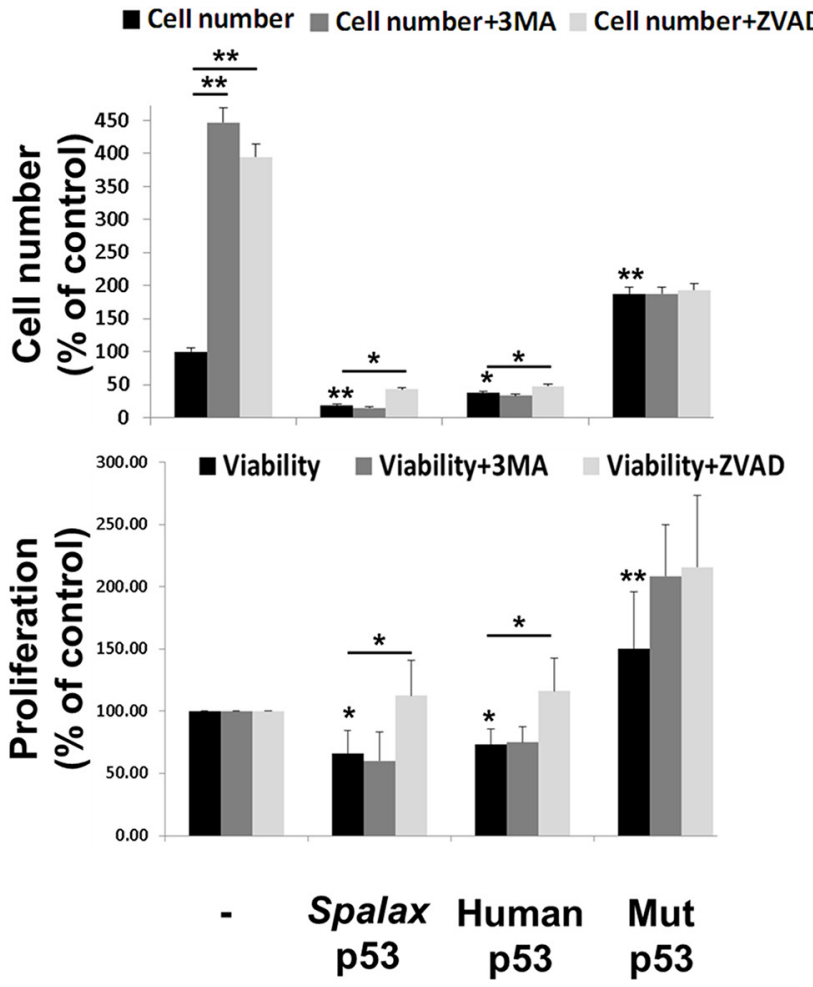

Figure 5: Lung cancer cell proliferation reduction by the Spalax p53 is caspase-dependent. (A) Microscopy images of H1299 cells 72 hours post transfections with Spalax p53, wild type human p53 and mutated H179R human p53 (Mut p53). H1299 cells were transfected with the various p53 vectors in the presence/absence of an apoptosis inhibitor (Z-VAD-FMK) or authophagy inhibitor (3MA) and examined for (B) Absolute cell number (FACS) and (C) WST-1 proliferation assay. Cell counts were repeated twice, WST-1 experiments were repeated three times in duplicates. Results are presented as $\%$ of empty vectors \pm inhibitors (pCMV for the human wild type and mutated p53; pCDNA3 for the Spalax p53). ${ }^{*} p<0,05, * * p<0.005$. 
by depleting endogenous inhibitors of these cell death pathways [43]. This further suggests that, even though apoptosis is part of the mechanisms through which the cells are finally destroyed, autophagy can also mediate cell elimination as a genuine effector mechanism [40]. As p53 was shown to transcriptionally activate several target genes that result in the activation of autophagy [43, 46], assessments of a similar action by the Spalax p53 is merited.

To conclude the blind more rat, Spalax, shows a striking resistance to cancer. Our recent findings suggest that this resistance phenotype may be partly mediated by Spalax's unique p53 protein, which seems to retain potent and efficient cancer death activities, which are mainly autophagy mediated and caspase-dependent.

\section{MATERIALS AND METHODS}

\section{Plasmids}

For transfection studies, we have used expression plasmids of the human p53, human mutated p53 (H179R), and Spalax p53, as previously described $[6,8]$. The human wild-type p53 was ligated into a pCMV (cytomegalovirus) construct. Spalax p53 cDNA was cloned from mRNA prepared from a whole embryo belonging to the S. judaei superspecies and was ligated into a pCDNA3 expression vector. Empty vectors were used appropriately for normalization in all experiments.

\section{Reagents and chemicals}

Pan-caspase specific apoptosis inhibitor (Z-VADFMK, ALX-260-020-M001, Enzo Life Sciences). Authophagy inhibitor (3MA), Bafilomycin A1 (B1793) and Acridine Orange (A6014) from Sigma Aldrich. 30\% Hydrogen peroxide $\left(\mathrm{H}_{2} \mathrm{O}_{2}\right)$ was from Merck Millipore (107209).

\section{Cell culture}

H1299 cells (human non-small cell lung cancer, ATCC HTB-96) were grown in 10\% FCS in RPMI medium 1640 (Sigma) supplemented with $2 \mathrm{mM}$ glutamine, $100 \mu \mathrm{g} / \mathrm{ml}$ streptomycin, and 100 units $/ \mathrm{ml}$ penicillin at $37^{\circ} \mathrm{C}$ in a humidified incubator with $5 \%$ $\mathrm{CO}_{2}$. The cells were tested negative for mycoplasma contamination using the e-Myco PCR mycoplasma detection kit (25235, iNtRON Biothechnology).

\section{Transient transfections}

H1299 cell lines were plated $\left(2 \times 10^{4} / 96\right.$ well plates). Transient transfection was performed the next day using JetPEI transfection reagent (101-10 N ) according to manufacturer's instructions (Polyplus Transfection). Cells were transfected with $1 \mu \mathrm{g}$ of the various $p 53$ plasmids in the presence/absence of pan-caspase specific apoptosis inhibitor (50 $\mu \mathrm{M} Z$ Z-VAD-FMK) or authophagy inhibitor (10 mM, 3MA). Appropriate controls were performed for each assay and included vehicle only and transfections with empty vectors (pCDNA3 or pCMV) in the presence/ absence of the apoptosis or authophagy inhibitors. Cell were analyzed by the methods detailed below, 72 hours post transfections.

\section{Flow cytometry}

MACSQuant (Miltenyi Biotec) was used. For absolute cell number, the cells were harvested 72 hours post trasnfections, in a fixed volume and counted. For annexin-PI assay, cells were harvested 72 hours post trasnfections and incubated with Annexin v-FITC and PI (K101-400, BioVision Inc.) according to manufacturer's instructions. Annexin-/PI-, surviving cell fraction; annexin+/PI-, early apoptosis; and annexin+/PI+, late apoptosis/necrosis. For cell cycle analysis the cells were harvested, 72 hours post trasnfections, permeabilized by $70 \%$ ethanol for $20 \mathrm{~min}$ at $-20^{\circ} \mathrm{C}$ and stained for 15 min at room temperature with DNA propidium iodide (PI) $(50 \mu \mathrm{g} / \mathrm{mL}) / \mathrm{RNAse} A(10 \mu \mathrm{g} / \mathrm{mL})$ (P4170, SigmaAldrich).

\section{Proliferation assay}

WST-1 (Roche, Indianapolis, IN, USA; 10\% final concentration) was incubated with cells at $37{ }^{\circ} \mathrm{C}$ for $1 \mathrm{~h}$ and read with a microELISA reader at $440 \mathrm{~nm}$.

\section{Supravital cell-staining with acridine orange for autophagy detection}

Acridine orange was added at a final concentration of $1 \mu \mathrm{g} / \mathrm{ml}$ for a period of 15 minutes to the cells, 72 hours post trasnfections. Bafilomycin A1, an autophagy inhibitor, dissolved in DMSO, was added to the cells 30 min before addition of acridine orange. Appropriate negative (empty vectors) or positive controls (cells treated with $3 \% \mathrm{H}_{2} \mathrm{O}_{2}$ ) were done. The cells were then washed twice with PBS. Pictures were obtained with a fluorescence microscope. Fluorescence was quantified using NIH ImageJ software.

\section{Quantification of GFP-LC3 levels}

The turnover of GFP-LC3 (reflecting an autophagic flux) is a reliable and simple assay to measure autophagic activity in living mammalian cells [47]. Enhanced autophagic flux is detected as a decreased total cellular GFP signal. GFP fused to the $\mathrm{N}$ terminus of LC3 (a generous gift from Prof. Zvulun Elazar, The Department of Biological Chemistry, The Weizmann Institute of Science, Rehovot, Israel). The GFP-LC3 plasmid was 
transfected in the presence/absence of the various p53 plasmids, as described above. The levels of GFP in the cells was quantified 72 hours post transfection, by flowcytometry (MACSQuant).

\section{Light and fluorescent microscopy}

The cells were visualized, 72 hours post trasnfections, by a light and fluorescence microscope equipped with a camera (model IX71; Olympus) with a $\times$ 20/0.50 objective lens and $\mathrm{Cell}^{\wedge} \mathrm{A}$ (version 3.1) Olympus software imaging. Fluorescence was quantified using NIH ImageJ software.

\section{Statistical analysis}

Experiments were analyzed by a two-sided Student's unpaired $t$-test for significance $(P<0.05)$.

\section{CONFLICTS OF INTEREST}

None to disclose.

\section{REFERENCES}

1. Nevo E, Beiles A, Spradling T. Molecular evolution of cytochrome b of subterranean mole rats, Spalax ehrenbergi superspecies, in Israel. J Mol Evol. 1999; 49:215-226.

2. Nevo E. Evolution of genome-phenome diversity under environmental stress. Proc Natl Acad Sci U S A. 2001; 98:6233-6240.

3. Avivi A, Resnick MB, Nevo E, Joel A, Levy AP. Adaptive hypoxic tolerance in the subterranean mole rat Spalax ehrenbergi: the role of vascular endothelial growth factor. FEBS Lett. 1999; 452:133-140.

4. Wang X, Simpson ER, Brown KA. p53: Protection against Tumor Growth beyond Effects on Cell Cycle and Apoptosis. Cancer Res. 2015; 75:5001-5007.

5. Kruiswijk F, Labuschagne CF, Vousden KH. p53 in survival, death and metabolic health: a lifeguard with a licence to kill. Nat Rev Mol Cell Biol. 2015; 16:393-405.

6. Ashur-Fabian O, Avivi A, Trakhtenbrot L, Adamsky K, Cohen M, Kajakaro G, Joel A, Amariglio N, Nevo E, Rechavi G. Evolution of p53 in hypoxia-stressed Spalax mimics human tumor mutation. Proc Natl Acad Sci U S A. 2004; 101:12236-12241.

7. Avivi A, Ashur-Fabian O, Joel A, Trakhtenbrot L, Adamsky K, Goldstein I, Amariglio N, Rechavi G, Nevo E. P53 in blind subterranean mole rats--loss-of-function versus gain-of-function activities on newly cloned Spalax target genes. Oncogene. 2007; 26:2507-2512.

8. Avivi A, Ashur-Fabian O, Amariglio N, Nevo E, Rechavi G. p53-a key player in tumoral and evolutionary adaptation: a lesson from the Israeli blind subterranean mole rat. Cell Cycle. 2005; 4:368-372.
9. Zhao Y, Tang JW, Yang Z, Cao YB, Ren JL, Ben-Abu Y, Li K, Chen XQ, Du JZ, Nevo E. Adaptive methylation regulation of $\mathrm{p} 53$ pathway in sympatric speciation of blind mole rats, Spalax. Proc Natl Acad Sci U S A. 2016; 113:2146-2151.

10. Tsujimoto Y, Shimizu S. Another way to die: autophagic programmed cell death. Cell Death Differ. 2005; 12: 1528-1534.

11. Zhao Y, Qu T, Wang P, Li X, Qiang J, Xia Z, Duan H, Huang J, Zhu L. Unravelling the relationship between macroautophagy and mitochondrial ROS in cancer therapy. Apoptosis. 2016; 21:517-531.

12. Manov I, Hirsh M, Iancu TC, Malik A, Sotnichenko N, Band M, Avivi A, Shams I. Pronounced cancer resistance in a subterranean rodent, the blind mole-rat, Spalax: in vivo and in vitro evidence. BMC Biol. 2013; 11:91.

13. Kenzelmann Broz D, Spano Mello S, Bieging KT, Jiang D, Dusek RL, Brady CA, Sidow A, Attardi LD. Global genomic profiling reveals an extensive p53-regulated autophagy program contributing to key p53 responses. Genes Dev. 2013; 27:1016-1031.

14. Avivi A, Shams I, Joel A, Lache O, Levy AP, Nevo E. Increased blood vessel density provides the mole rat physiological tolerance to its hypoxic subterranean habitat. Faseb J. 2005; 19:1314-1316.

15. Shams I, Avivi A, Nevo E. Hypoxic stress tolerance of the blind subterranean mole rat: expression of erythropoietin and hypoxia-inducible factor 1 alpha. Proc Natl Acad Sci U S A. 2004; 101:9698-9703.

16. Shams I, Nevo E, Avivi A. Ontogenetic expression of erythropoietin and hypoxia-inducible factor-1 alpha genes in subterranean blind mole rats. Faseb J. 2005; 19:307-309.

17. Shams I, Avivi A, Nevo E. Oxygen and carbon dioxide fluctuations in burrows of subterranean blind mole rats indicate tolerance to hypoxic-hypercapnic stresses. Comp Biochem Physiol A Mol Integr Physiol. 2005; 142:376-382.

18. Schmidt H, Hangmann J, Shams I, Avivi A, Hankeln T. Molecular evolution of antioxidant and hypoxia response in long-lived, cancer-resistant blind mole rats: The Nrf2Keap1 pathway. Gene. 2016; 577:293-298.

19. Kroemer G, Jaattela M. Lysosomes and autophagy in cell death control. Nat Rev Cancer. 2005; 5:886-897.

20. Maiuri MC, Tasdemir E, Criollo A, Morselli E, Vicencio JM, Carnuccio R, Kroemer G. Control of autophagy by oncogenes and tumor suppressor genes. Cell Death Differ. 2009; 16:87-93.

21. White E. Autophagy and p53. Cold Spring Harb Perspect Med. 2016; 6.

22. Band M, Joel A, Hernandez A, Avivi A. Hypoxia-induced BNIP3 expression and mitophagy: in vivo comparison of the rat and the hypoxia-tolerant mole rat, Spalax ehrenbergi. Faseb J. 2009; 23:2327-2335.

23. Rodriguez KA, Li K, Nevo E, Buffenstein R. Mechanisms regulating proteostasis are involved in sympatric speciation 
of the blind mole rat, Spalax galili. Autophagy. 2016; 12:703-704.

24. Rodriguez KA, Valentine JM, Kramer DA, Gelfond JA, Kristan DM, Nevo E, Buffenstein R. Determinants of rodent longevity in the chaperone-protein degradation network. Cell Stress Chaperones. 2016.

25. Band M, Ashur-Fabian O, Avivi A. The expression of p53target genes in the hypoxia-tolerant subterranean mole-rat is hypoxia-dependent and similar to expression patterns in solid tumors. Cell Cycle. 2010; 9:3347-3352.

26. Fang X, Nevo E, Han L, Levanon EY, Zhao J, Avivi A, Larkin D, Jiang X, Feranchuk S, Zhu Y, Fishman A, Feng Y, Sher N, et al. Genome-wide adaptive complexes to underground stresses in blind mole rats Spalax. Nat Commun. 2014; 5:3966.

27. Shams I, Malik A, Manov I, Joel A, Band M, Avivi A. Transcription pattern of $\mathrm{p} 53$-targeted DNA repair genes in the hypoxia-tolerant subterranean mole rat Spalax. J Mol Biol. 2013; 425:1111-1118.

28. Haupt Y, Barak Y, Oren M. Cell type-specific inhibition of p53-mediated apoptosis by mdm2. Embo J. 1996; 15: 1596-1606.

29. Chen X, Ko LJ, Jayaraman L, Prives C. p53 levels, functional domains, and DNA damage determine the extent of the apoptotic response of tumor cells. Genes Dev. 1996; 10:2438-2451.

30. Kagawa S, Fujiwara T, Hizuta A, Yasuda T, Zhang WW, Roth JA, Tanaka N. p53 expression overcomes p21WAF1/ CIP1-mediated G1 arrest and induces apoptosis in human cancer cells. Oncogene. 1997; 15:1903-1909.

31. Solomon H, Buganim Y, Kogan-Sakin I, Pomeraniec L, Assia Y, Madar S, Goldstein I, Brosh R, Kalo E, Beatus T, Goldfinger N, Rotter V. Various p53 mutant proteins differently regulate the Ras circuit to induce a cancerrelated gene signature. J Cell Sci. 2012; 125:3144-3152.

32. Kagan VE, Fabisiak JP, Shvedova AA, Tyurina YY, Tyurin VA, Schor NF, Kawai K. Oxidative signaling pathway for externalization of plasma membrane phosphatidylserine during apoptosis. FEBS Lett. 2000; 477:1-7.

33. Petrovski G, Zahuczky G, Katona K, Vereb G, Martinet W, Nemes Z, Bursch W, Fesus L. Clearance of dying autophagic cells of different origin by professional and non-professional phagocytes. Cell Death Differ. 2007; 14:1117-1128.

34. Qu X, Zou Z, Sun Q, Luby-Phelps K, Cheng P, Hogan RN, Gilpin C, Levine B. Autophagy gene-dependent clearance of apoptotic cells during embryonic development. Cell. 2007; 128:931-946.

35. Mellen MA, de la Rosa EJ, Boya P. Autophagy is not universally required for phosphatidyl-serine exposure and apoptotic cell engulfment during neural development. Autophagy. 2009; 5:964-972.

36. Lenardo MJ, McPhee CK, Yu L. Autophagic cell death. Methods Enzymol. 2009; 453:17-31.

37. Zhao Y, Ren JL, Wang MY, Zhang ST, Liu Y, Li M, Cao YB, Zu HY, Chen XC, Wu CI, Nevo E, Chen XQ, $\mathrm{Du}$ JZ. Codon 104 variation of p53 gene provides adaptive apoptotic responses to extreme environments in mammals of the Tibet plateau. Proc Natl Acad Sci U S A. 2013; 110:20639-20644.

38. Mancias JD, Kimmelman AC. Mechanisms of Selective Autophagy in Normal Physiology and Cancer. J Mol Biol. 2016.

39. Galluzzi L, Vicencio JM, Kepp O, Tasdemir E, Maiuri MC, Kroemer G. To die or not to die: that is the autophagic question. Curr Mol Med. 2008; 8:78-91.

40. Maiuri MC, Zalckvar E, Kimchi A, Kroemer G. Self-eating and self-killing: crosstalk between autophagy and apoptosis. Nat Rev Mol Cell Biol. 2007; 8:741-752.

41. Miki H, Uehara N, Kimura A, Sasaki T, Yuri T, Yoshizawa K, Tsubura A. Resveratrol induces apoptosis via ROS-triggered autophagy in human colon cancer cells. Int J Oncol. 2012; 40:1020-1028.

42. Wu H, Che X, Zheng Q, Wu A, Pan K, Shao A, Wu Q, Zhang J, Hong Y. Caspases: a molecular switch node in the crosstalk between autophagy and apoptosis. Int J Biol Sci. 2014; 10:1072-1083.

43. Marino G, Niso-Santano M, Baehrecke EH, Kroemer G. Self-consumption: the interplay of autophagy and apoptosis. Nat Rev Mol Cell Biol. 2014; 15:81-94.

44. Gonzalez-Polo RA, Boya P, Pauleau AL, Jalil A, Larochette N, Souquere S, Eskelinen EL, Pierron G, Saftig P, Kroemer G. The apoptosis/autophagy paradox: autophagic vacuolization before apoptotic death. J Cell Sci. 2005; 118:3091-3102.

45. Siedlecka-Kroplewska K, Jozwik A, Boguslawski W, Wozniak M, Zauszkiewicz-Pawlak A, Spodnik JH, Rychlowski M, Kmiec Z. Pterostilbene induces accumulation of autophagic vacuoles followed by cell death in HL60 human leukemia cells. J Physiol Pharmacol. 2013; 64:545-556.

46. Feng $\mathrm{Z}$, Zhang $\mathrm{H}$, Levine AJ, Jin S. The coordinate regulation of the $\mathrm{p} 53$ and $\mathrm{mTOR}$ pathways in cells. Proc Natl Acad Sci U S A. 2005; 102:8204-8209.

47. Shvets E, Elazar Z. Flow cytometric analysis of autophagy in living mammalian cells. Methods Enzymol. 2009; 452:131-141. 